\title{
环庚三烯酮衍生物的合成和抑制胃癌细胞增殖的活性研究
}

\author{
郡继炎†, $a$ 李志杰†, $a$ 王连君 $b$ 熊裕炎 $*, b$ 胡向东 $*, a$ \\ ( ${ }^{a}$ 西北大学化学与材料科学学院 合成与天然功能分子教育部重点实验室 西安 710127) \\ ( $b$ 西北大学医学院 西部资源生物与现代生物技术教育部重点实验室 西安 710069)
}

\begin{abstract}
摘要 基于已知的金催化氧化六元环扩环策略, 实现了一系列环庚三烯酮衍生物的合成. 发现环庚三烯酮衍生物 2-羟 基-3,6-二甲基-5-氧代-1,3,6-环庚三烯-1-羧酸乙酯(3)、3,6-二甲基-7-氧代-4-((三氟甲基)磺酰基)氧基)-1,3,5-环庚三烯-1羧酸乙酯(9)、3,6-二甲基-7-氧代-4-乙烯基-1,3,5-环庚三烯-1-羧酸乙酯(10)人胃癌细胞 MGC-27 表现出抗增殖活性, 而活 性相对较好的化合物 9 的抗增殖活性与胃癌细胞 MGC-27 调亡没有关联.

关键词＼cjkstart环庚三烯酮衍生物；七元芳环；胃癌；生物活性
\end{abstract}

\section{Synthesis and Bioactivity of Tropone Derivatives as Potential Compounds against Human Gastric Cancer Cells Growth}

\author{
Shao, Jiyan ${ }^{\dagger, a} \quad$ Li, Zhiji ${ }^{\dagger, a} \quad$ Wang, Yajun $^{b} \quad$ Xiong, Yuyan $^{*, b} \quad \mathrm{Hu}$, Xiangdong ${ }^{*, a}$ \\ $\left({ }^{a}\right.$ Key Laboratory of Synthetic and National Functional Molecule Chemistry of Ministry of Education, \\ College of Chemistry \& Materials Science, Northwest University, Xi'an 710127) \\ ( ${ }^{b}$ Key Laboratory of Resource Biology and Biotechnology in Western China, Ministry of Education, \\ School of Medicine, Northwest University, Xi'an 710069)
}

\begin{abstract}
Based on the strategy of Au-catalyzed oxidative ring expansion of six-membered rings, the synthesis of a series of tropone derivatives was achieved. It was disclosed that tropone derivatives ethyl 2-hydroxy-3,6-dimethyl-5-oxocyclohepta1,3,6-triene-1-carboxylate (3), ethyl 3,6-dimethyl-7-oxo-4-(((trifluoromethyl)sulfonyl)oxy)cyclohepta-1,3,5-triene-1-carboxylate (9), and ethyl 3,6-dimethyl-7-oxo-4-vinylcyclohepta-1,3,5-triene-1-carboxylate (10) displayed antiproliferative activity against human gastric cancer cells MGC-27. Compound 9, which possesses relatively better activity, didn't show relationship between its antiproliferative activity and the apoptosis of gastric cancer cells MGC-27.

Keywords tropone; seven-membered aromatic ring; gastric cancer; bioactivity
\end{abstract}

环庚三烯酮(tropone, 图 1)是一种存在于多种天然 产物中的芳香性化合物 ${ }^{[1-3]}$. 由于具有独特的分子结 构[4-6]、丰富的化学性质 ${ }^{[7-13]}$ 和重要的生理活性 ${ }^{[14-25]}$, 环 庚三烯酮类化合物得到了有机化学家的广泛关注. 例如 桧木醇(hinokitiol)拥有抗菌以及抑制黑色素生成的活性, 具有一定的皮肤美白作用 ${ }^{[26-27]}$. Pareitropone 对白血病 P388 细胞表现出很强的细胞毒性 ${ }^{[28-30]}$, 秋水仙碱 (colchicine) 被作为药物用于痛风和多种癌症的治

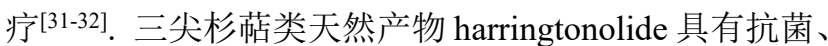
抗病毒、抗癌等多种显著的生物活性 ${ }^{[33-35]}$. 此外, 一些 人工合成的环庚三烯酮衍生物也表现出良好的生物活 性. 如 Yamato 课题组 ${ }^{[36-40]}$ 报道的非天然环庚三烯酮衍 生物表现出抑制肿瘤细胞生长的活性.

胃癌是一种常见的消化系统恶性肿瘤，其致死率占 据全球癌症疾病第二位 ${ }^{[41-44]}$. 在许多亚洲国家，胃癌的 发生率仍然居高不下 ${ }^{[45-46]}$. 因此，针对胃癌治疗的先导

\footnotetext{
* Corresponding authors. E-mail: yuyan.xiong@nwu.edu.cn; xiangdonghu@nwu.edu.cn Received May 10, 2021; revised May 31, 2021; published online June 15, 2021.

Project supported by the National Science Foundation of China (Nos. 21772153, 22071192), the Science and Technology Department of Shaanxi Province (No. 2019JM-151), the Key Science and Technology Innovation Team of Shaanxi Province (No. 2017KCT-37), the Natural Science Foundation of Shaanxi Province (No. 2020JQ-611), the Special Scientific Research Plan for Emergency Public Health Safety 2020 of Shaanxi Provincial Department of Education (No. 20JG034) and the High-level Talent Programs of Shaanxi Province.

国家自然科学基金(Nos. 21772153, 22071192)、陕西省科技厅基金(No. 2019JM-151)、陕西省科技创新团队(No. 2017KCT-37)、陕西省自然科学基金(No. 2020JQ-611)、陕西省教育厅 2020 年突发公共卫生安全专项科学研究计划(No. 20JG034)和陕西省高层次人才资助项目.

$\dagger$ 共同第一作者(These authors contributed equally to this work).
} 
化合物研究是一个颇有意义的研究课题. 本文设计合成 了一系列带有不同官能团的环庚三烯酮类化合物, 并进 行了初步的生理活性研究. 结果显示部分化合物具有明 显的胃癌细胞抗增殖活性.

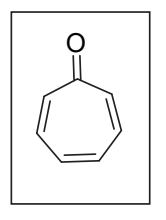

tropone<smiles>COc1cc2ccnc3c2c(c1OC)-c1ccc(=O)ccc1-3</smiles>

pareitropone

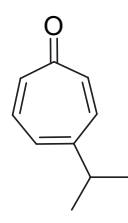

nezukone<smiles>COc1cc2c(c(OC)c1OC)-c1ccc(OC)c(=O)cc1[C@@H](NC=O)CC2</smiles>

colchicine

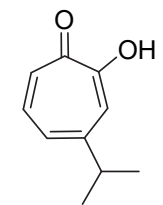

hinokitiol

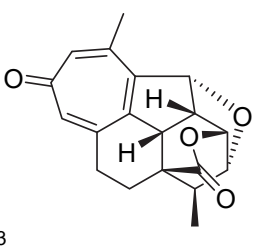

harringtonolide
图 1 环庚三烯酮及其相关天然产物

Figure 1 Tropone and representative natural products with tropone moiety

\section{1 结果和讨论}

对环庚三烯酮的合成已有许多已知方法. 包括七元 环化合物的直接氧化、二烯化合物的关环复分解 ${ }^{[47-50]}$ 、 六元环扩环反应、环加成反应等 ${ }^{[51-56]}$. 本文采用了刘元 红课题组 ${ }^{[57-59]}$ 报道的六元环扩环策略.

首先, 以 2,5-二甲基-1,4-苯醌(1)为起始原料, 经其 与乙氧基乙炔锂的 1,2-加成反应得到化合物 2 (Scheme 1). 然后研究了化合物 2 的金催化氧化扩环反应, 在 $\mathrm{PPh}_{3} \mathrm{AuTf}_{2}$ 为催化剂、吡定氮氧化物为氧化剂的条件下, 化合物 2 顺利实现了扩环反应，以 $72 \%$ 的产率生成了化 合物 3. 接着, 化合物 3 在碱性条件下和 MeI 反应, 得到 了两个甲基化的产物 5 和 $\mathbf{6}$, 这主要是因为在碱性条件 下化合物 3 和其异构化产物 4 可以互相转化 ${ }^{[57]}$. 通过单 晶衍射确定了化合物 6 的结构(CCDC 2071186, 图 2). 有趣的是, 当化合物 3 与 $\mathrm{PCl}_{3}$ 在二氯乙烷中反应时, 得 到了单一的氯代产物 7; 而化合物 3 在与 $N$-溴代丁二酰 亚胺(NBS)反应时，得到了化合物 8.

随后, 将化合物 3 和三氟甲磺酸酐在低温条件下反 应, 得到了单一的三氟甲磺酰化产物 9 (Scheme 2). 尝 试了化合物 7、8、9 与三丁基乙烯基锡的 Stille 偶联反 应，发现化合物 7 不能发生相应的偶联反应，化合物 9 能以 $50 \%$ 的产率给出相应产物 $\mathbf{1 0}$, 化合物 $\mathbf{8}$ 虽能反应但 产率并不理想. 在对化合物 9 中末端双键转为成醛基的 尝试中, 首先使用了 $\mathrm{RuCl}_{3} \cdot 3 \mathrm{H}_{2} \mathrm{O} / \mathrm{PhI}(\mathrm{OAc})_{2}$, 但只能以 $20 \%$ 的产率得到 11 . 经过条件篮选, 发现 $\mathrm{K}_{2} \mathrm{OsO}_{4} \cdot 2 \mathrm{H}_{2} \mathrm{O} /$ $\mathrm{NaIO}_{4}$ 能够顺利地实现化合物 $\mathbf{1 0}$ 的 “一锅法” 双羟化/
氧化切断，生成预期的产物 11，产率为 $75 \%$. 化合物 11 再经 $\mathrm{Bu}_{4} \mathrm{NBH}_{4}$ 还原得到醇 12. 化合物 12 经过乙酰化生 成环庚三烯酮衍生物 13, 其分子结构经过单晶衍射得 以确认(CCDC 2071193, 图 2).

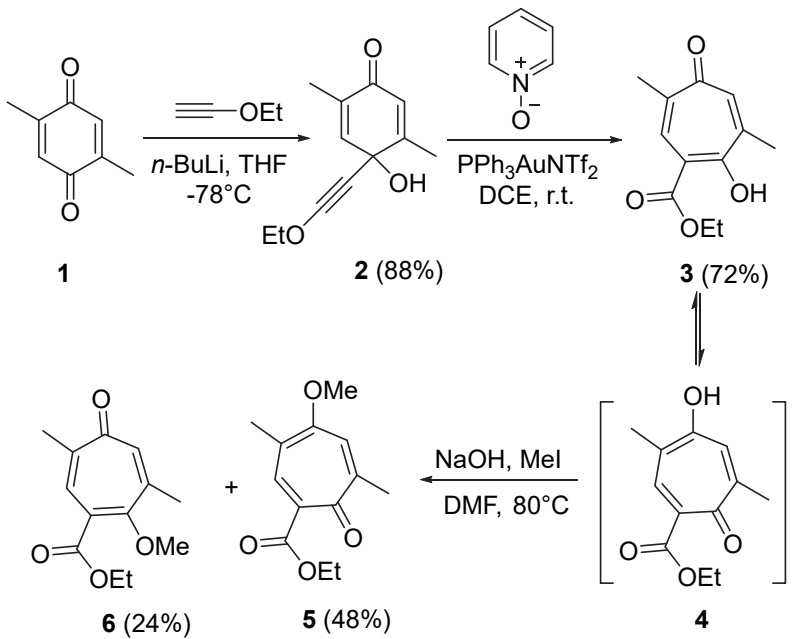

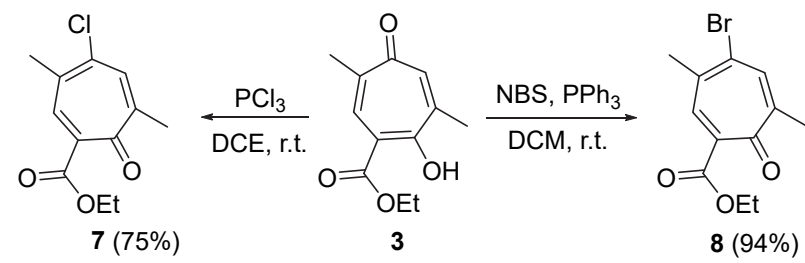

图式 1 环庚三烯酮衍生物的合成

Scheme 1 Synthesis of tropones derivatives.

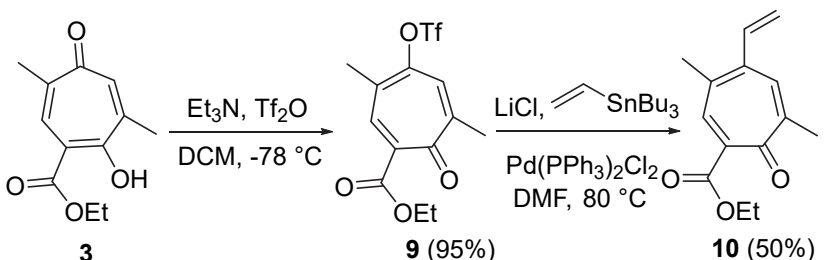

3 $9(95 \%)$ $10(50 \%)$ $\mathrm{NaIO}_{4}, \mathrm{~K}_{2} \mathrm{OsO}_{4} \cdot 2 \mathrm{H}_{2} \mathrm{O}$
2,6 -lutidine
$V($ acetone $): V\left(\mathrm{H}_{2} \mathrm{O}\right)=3: 1$

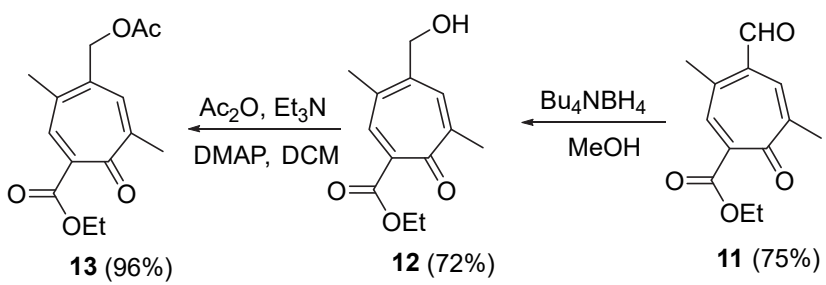

图式 2 环庚三烯酮衍生物 9 13 的合成

Scheme 2 Synthesis of tropone derivatives $9 \sim 13$

对合成得到的环庚三烯酮衍生物 $3 、 5 \sim 13$ 的生物 活性进行了研究. 如图 3 所示, 在对胃癌细胞 MGC-27 的存活抑制实验中，化合物 3、9、10 表现出良好的抑制 活性，而其它化合物的抑制能力相对较弱. 


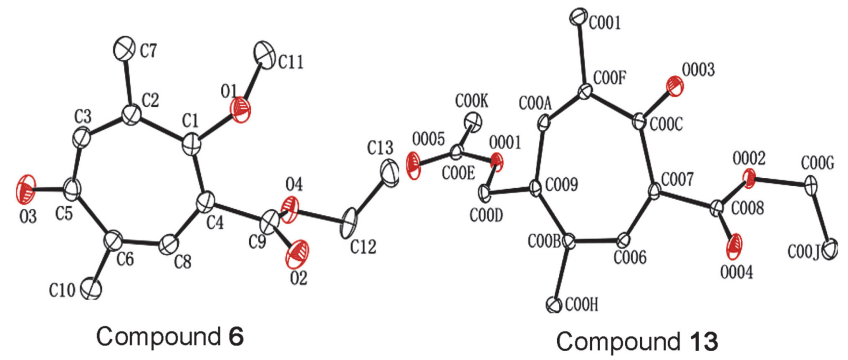

图 2 环庚三烯酮衍生物 $\mathbf{6}$ 和 13 的晶体结构

Figure 2 Crystal structure of tropones derivatives $\mathbf{6}$ and $\mathbf{1 3}$

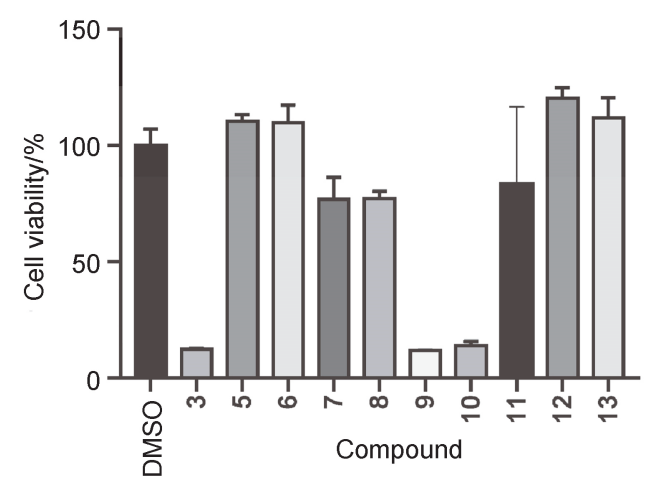

图 3 环庚三烯酮衍生物 3、5 13 对人胃癌细胞 MGC-27 细 胞存活率的影响(DMSO 作为空白对照)

Figure 3 Effects of tropone derivatives 3, 5 13 on the cell viability of human gastric cancer cells MGC-27 (DMSO was used as negative control)

随后研究活性较好的化合物 9 对胃癌细胞 MGC-27 的生长抑制曲线(图 4), 得出 $50 \%$ 抑制浓度 $\left(\mathrm{GI}_{50}\right)$ 值为 $3.912 \mu \mathrm{mol} / \mathrm{L}$.

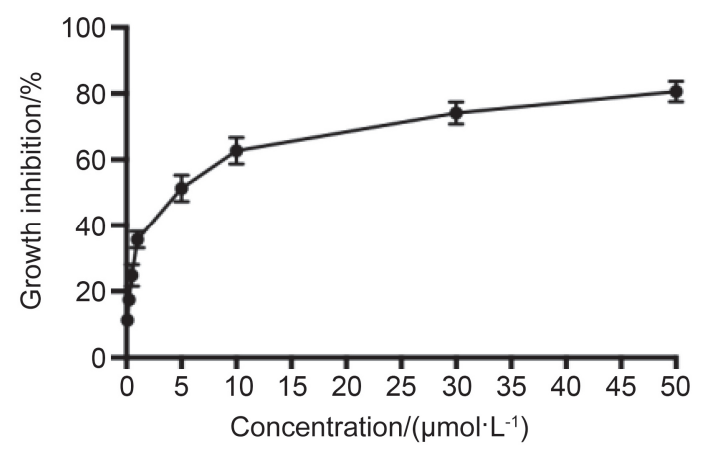

图 4 环庚三烯酮衍生物 9 对人胃癌细胞 MGC-27 的生长抑制 曲线

Figure 4 Growth inhibition curve of tropone derivative 9 in human gastric cancer cells MGC-27

为了初步了解环庚三烯酮衍生物 9 对胃癌细胞 MGC-27 生长抑制的可能机理, 采用膜联蛋白(V-FITC) 细胞调亡检测试剂盒的流式细胞术检测了化合物 9 对胃 癌细胞 MGC-27 细胞调亡的影响. 如图 5 所示, 与对照 组(DMSO)相比, 化合物 9 没有明显促进 MGC-27 细胞
调亡的表现. 这些结果表明，化合物 9 对胃癌细胞 MGC-27 的生成抑制活性可能是通过抑制该细胞的增殖 而不是诱导该细胞调亡的途径来实现的. 关于其详细的 作用机制仍需进一步的深入研究.
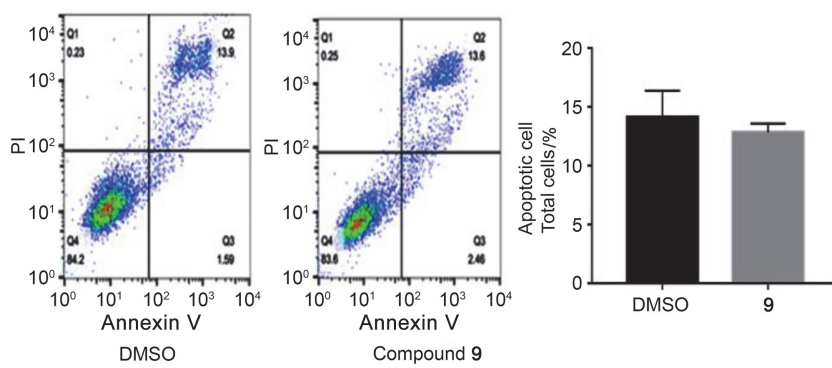

图 5 化合物 9 对人胃癌细胞 MGC-27 调亡的影响

Figure 5 Effects of compound 9 on the apoptosis of human gastric cancer cells MGC-27

\section{2 结论}

基于金催化氧化六元环扩环反应，成功合成了一系 列官能团化的环庚三烯酩衍生物, 并就这些化合物对胃 癌细胞 MGC-27 的抗增殖活性进行了初步研究. 结果表 明环庚三烯酮衍生物 9 对胃癌细胞 MGC-27 表现出突出 的抗增殖活性, 具有良好的深入研究价值.

\section{3 实验部分}

\section{1 仪器与试剂}

${ }^{1} \mathrm{H}$ NMR 和 ${ }^{13} \mathrm{C}$ NMR 用 BRUKER-ASCEND 400 $\mathrm{MHz}$ 核磁共振仪和日本电子 JEOL $400 \mathrm{YH}$ 核磁共振仪 测定, 以 $\mathrm{CDCl}_{3}$ 为溶剂, $\mathrm{TMS}$ 为内标; 高分辨质谱用 BRUKER micrOTOF-QII \& Thermo Ultimate3000 质谱仪 测定. 未有特殊说明, 所有反应均在干燥氮气保护下进 行. 无水溶剂按照常规方法处理和使用, 其它试剂均为 分析纯.

\section{2 实验方法}

3.2.14-(乙氧乙炔基)-4-羟基-2,5-二甲基-2,5-环已二 烯-1-酮(2)的合成

将乙氧基乙炔 $(45 \%$ 的己烷溶液, $1.47 \mathrm{~g}, 9.50 \mathrm{mmol})$ 溶于无水四氢呋喃 $(20 \mathrm{~mL})$ 中, 将反应体系冷冻至一 $78{ }^{\circ} \mathrm{C}$, 滴加 $n$-BuLi $\left(2.5 \mathrm{~mol} \cdot \mathrm{L}^{-1}\right.$ 的已烷溶液, $3.32 \mathrm{~mL}$, $8.10 \mathrm{mmol})$. 在此温度下搅拌 $1 \mathrm{~h}$ 后, 加入化合物 $\mathbf{1}(1 \mathrm{~g}$, $7.34 \mathrm{mmol}$ ), 在相同温度下搅拌 $3 \mathrm{~h}$. 原料消耗完毕后, 用饱和 $\mathrm{NH}_{4} \mathrm{Cl}$ 溶液淬灭. 分离有机相, 水相用乙酸乙酯 萃取 $(10 \mathrm{~mL} \times 3)$. 合并有机相, 用饱和食盐水洗涤, 无 水 $\mathrm{Na}_{2} \mathrm{SO}_{4}$ 干燥后浓缩, 硅胶柱色谱法纯化 [ $V$ (石油醚) : $V($ 乙酸乙酯 $)=10 ： 1$ 得到目标产物 2 . 棕褐色的油状液 体, $1.32 \mathrm{~g}$, 产率 $87.4 \% .{ }^{1} \mathrm{H}$ NMR $\left(400 \mathrm{MHz}, \mathrm{CDCl}_{3}\right) \delta$ : $6.66 \sim 6.59(\mathrm{~m}, 1 \mathrm{H}), 5.95(\mathrm{q}, J=1.8 \mathrm{~Hz}, 1 \mathrm{H}), 4.17 \sim 4.08$ 
$(\mathrm{m}, 2 \mathrm{H}), 2.76 \sim 2.47(\mathrm{~m}, 1 \mathrm{H}), 2.13(\mathrm{~d}, J=1.7 \mathrm{~Hz}, 3 \mathrm{H})$, $1.85(\mathrm{~d}, J=2.0 \mathrm{~Hz}, 3 \mathrm{H}), 1.40 \sim 1.32(\mathrm{~m}, 3 \mathrm{H}) ;{ }^{13} \mathrm{C} \mathrm{NMR}$ $\left(100 \mathrm{MHz}, \mathrm{CDCl}_{3}\right) \delta: 186.50,157.85,144.14,132.08$, $124.87,93.75,75.41,65.14,37.43,18.45,15.23,14.49$; IR (film) $v: 3356,2980,2923,2254,1675,1621,1433,1375$, 1289, 1241, 1193, 1150, 1090, 890, 824, 803, 775, 691 $\mathrm{cm}^{-1}$. HRMS $(\mathrm{ESI}+)$ calcd for $\mathrm{C}_{10} \mathrm{H}_{10} \mathrm{O}_{2}[\mathrm{M}+\mathrm{H}]^{+}$: 162.0675 , found 162.0658 .

3.2 .2 -羟基-3,6-二甲基-5-氧代-1,3,6-环庚三烯-1羧酸乙酯(3)的合成

将化合物 2 (1.32 g, $6.40 \mathrm{mmol})$ 溶于无水二氯乙烷 $(13 \mathrm{~mL})$ 中, 依次加入吡定氮氧化物(0.96 g, $7.68 \mathrm{mmol})$, $\mathrm{PPh}_{3} \mathrm{AuNTf}_{2}(0.117 \mathrm{mg}, 0.16 \mathrm{mmol})$, 室温搅拌 $3 \mathrm{~h}$. 薄层 色谱(TLC)监测原料消耗完毕后, 用 $5 \mathrm{~mL} \mathrm{NaOH}$ 溶液 $\left(0.5 \mathrm{~mol} \cdot \mathrm{L}^{-1}\right)$ 淬灭, 水相用二氯甲烷 $(\mathrm{DCM})$ 萃取, 然后 将 $5 \mathrm{~mL}$ 稀 $\mathrm{HCl}\left(1.0 \mathrm{~mol} \cdot \mathrm{L}^{-1}\right)$ 添加至水相, 有大量黄色 固体析出. 然后加入 DCM 溶解析出的固体, 所得混合 物用 $\mathrm{CH}_{2} \mathrm{Cl}_{2}$ 萃取 $(15 \mathrm{~mL} \times 3)$, 水洗涤直至 $\mathrm{pH}=7$. 有机 相用无水 $\mathrm{Na}_{2} \mathrm{SO}_{4}$ 干燥后浓缩, 硅胶柱色谱法纯化 $[V$ (石 油醚) $: V($ 乙酸乙酯 $)=10 ： 1]$ 得到目标产物 3 . 黄色固 体, $1.02 \mathrm{~g}$, 产率 $72 \%$. m.p. 138 $139{ }^{\circ} \mathrm{C} ;{ }^{1} \mathrm{H}$ NMR (400 $\left.\mathrm{MHz}, \mathrm{CDCl}_{3}\right) \delta: 14.14(\mathrm{~s}, 1 \mathrm{H}), 7.86(\mathrm{~s}, 1 \mathrm{H}), 7.17$ (s, 1H), 4.42 (q, $J=7.2 \mathrm{~Hz}, 2 \mathrm{H}), 2.29$ (s, 3H), 2.17 (s, 3H), 1.42 (t, $J=7.1 \mathrm{~Hz}, 3 \mathrm{H}) ;{ }^{13} \mathrm{C} \mathrm{NMR}\left(100 \mathrm{MHz}, \mathrm{CDCl}_{3}\right) \delta: 185.47$, $172.82,169.83,143.15,142.25,138.80,132.19,107.50$, 63.09, 22.72, 22.15, 14.21; IR (film) v: 3055, 2927, 1734, 1662, 1620, 1569, 1459, 1437, 1399, 1375, 1264, 1210, 994, 937, 895, $786 \mathrm{~cm}^{-1}$. HRMS $(\mathrm{ESI}+)$ calcd for $\mathrm{C}_{12} \mathrm{H}_{15} \mathrm{O}_{4}[\mathrm{M}+\mathrm{H}]^{+}:$223.0965, found 223.0964.

3.2.34-甲氧基-3,6-二甲基-7-氧代-1,3,5-环庚三烯1-羒酸乙酯(5)和 2-甲氧基-3,6-二甲基-5-氧代-1,3,6-环 庚三烯-1-羒酸乙酯 $(6)$ 的合成

将化合物 3 (200 mg, $0.90 \mathrm{mmol}$ ) 溶于无水 DMF (3 $\mathrm{mL})$ 中, 体系搅拌均匀后, 加入氢氧化钠 $(54 \mathrm{mg}, 1.35$ $\mathrm{mmol})$. 室温摚拌 $10 \mathrm{~min}$ 后滴入碘甲烷(320 mg, 2.25 $\mathrm{mmol}$ ), 加热至 $80{ }^{\circ} \mathrm{C}$ 下搅拌 $3 \mathrm{~h}$. TLC 监测原料基本消 耗完毕时, 用饱和 $\mathrm{NaHCO}_{3}$ 溶液淬灭. 分离有机相, 水 相用乙酸乙酯萃取 $(15 \mathrm{~mL} \times 3)$, 合并有机相, 用饱和食 盐水洗涤, 无水 $\mathrm{Na}_{2} \mathrm{SO}_{4}$ 干燥后浓缩, 硅胶柱色谱法纯化 $[V($ 石油醚 $): V($ 乙酸乙酯 $)=10 ： 1]$ 得到棕黄色油状液体 5 (100 mg, 48\%)和 6 (50 mg, 24\%).

4-甲氧基-3,6-二甲基-7-氧代-1,3,5-环庚三烯- 1-羧 酸乙酯(5): ${ }^{1} \mathrm{H}$ NMR (400 MHz, $\left.\mathrm{CDCl}_{3}\right) \delta: 7.54(\mathrm{~s}, 1 \mathrm{H})$, 7.28 (s, 1H), 4.36 (d, J=7.1 Hz, 2H), 3.92 (s, 3H), 2.39 (t, $J=0.9 \mathrm{~Hz}, 3 \mathrm{H}), 2.21(\mathrm{~s}, 3 \mathrm{H}), 1.36(\mathrm{t}, J=7.1 \mathrm{~Hz}, 3 \mathrm{H}) ;{ }^{13} \mathrm{C}$
NMR $\left(100 \mathrm{MHz}, \mathrm{CDCl}_{3}\right) \delta: 181.98,168.98,161.03$, $151.72,140.93,134.39,125.95,125.90,61.74,57.45$, 23.87, 19.87, 14.34; IR (film) v: 2982, 2847, 1720, 1674, $1595,1563,1501,1458,1388,1371,1301,1237,1176$, 1108, 1027, 985, 932, 889, 773, 701, 659, $627 \mathrm{~cm}^{-1}$. HRMS $(\mathrm{ESI}+)$ calcd for $\mathrm{C}_{13} \mathrm{H}_{17} \mathrm{O}_{4}[\mathrm{M}+\mathrm{H}]^{+}:$237.1121, found 237.1108 .

2-甲氧基-3,6-二甲基-5-氧代-1,3,6-环庚三烯-1-羧酸 乙酯(6): ${ }^{1} \mathrm{H}$ NMR (400 MHz, $\left.\mathrm{CDCl}_{3}\right) \delta: 7.16$ (q, $J=1.2$ $\mathrm{Hz}, 1 \mathrm{H}), 7.08$ (q, $J=1.1 \mathrm{~Hz}, 1 \mathrm{H}), 4.36$ (q, $J=7.2 \mathrm{~Hz}, 2 \mathrm{H})$, 3.74 (s, 3H), 2.27 (d, $J=1.2 \mathrm{~Hz}, 3 \mathrm{H}), 2.20$ (d, $J=1.2 \mathrm{~Hz}$, $3 \mathrm{H}), 1.37$ (t, $J=7.1 \mathrm{~Hz}, 3 \mathrm{H}) ;{ }^{13} \mathrm{C} \mathrm{NMR}\left(100 \mathrm{MHz}, \mathrm{CDCl}_{3}\right)$ $\delta: 185.48,167.98,160.74,147.63,144.14,140.51,132.16$, 128.12, 62.20, 22.10, 22.04, 14.27; IR (film) v: 2935, $1727,1622,1575,1510,1446,1375,1278,1212,1771$, 1212, 1171, 1119, 1020, 1000, 977, 861, 775, $722 \mathrm{~cm}^{-1}$. HRMS (ESI+) calcd for $\mathrm{C}_{13} \mathrm{H}_{17} \mathrm{O}_{4}[\mathrm{M}+\mathrm{H}]^{+}:$237.1121, found 237.1103 .

3.2.44-氯-3,6-二甲基-7-氧代-1,3,5-环庚三烯-1-羒 酸乙酯(7)的合成

将化合物 3 (60 mg, $0.28 \mathrm{mmol})$ 溶于二氯乙烷 $(3 \mathrm{~mL})$ 中, 然后加入三氯化磷(44 mg, $0.26 \mathrm{mmol})$, 室温搅拌 1 $\mathrm{h}, \mathrm{TLC}$ 监测原料消耗完毕后, 用 $\mathrm{NaHCO}_{3}$ 溶液淬灭. 分 离有机相, 水相用二氯甲烷萃取 $(5 \mathrm{~mL} \times 3)$, 合并有机相 并用饱和食盐水洗涤, 无水 $\mathrm{Na}_{2} \mathrm{SO}_{4}$ 干燥后浓缩, 硅胶柱 色谱法纯化 $[V$ (石油醚) : $V$ (乙酸乙酯 $)=10 ： 1$ 得到黄色 固体 7 (50 mg, 75\%). m.p. 52 54 ${ }^{\circ} \mathrm{C} ;{ }^{1} \mathrm{H}$ NMR (400 $\left.\mathrm{MHz}, \mathrm{CDCl}_{3}\right) \delta: 7.44$ (s, 1H), 7.39 (s, 1H), 4.34 (q, $J=7.1$ $\mathrm{Hz}, 2 \mathrm{H}), 2.40$ (s, 3H), $2.28(\mathrm{~s}, 3 \mathrm{H}), 1.34(\mathrm{t}, J=7.1 \mathrm{~Hz}$, $\left.3 \mathrm{H}) ;{ }^{13} \mathrm{C} \mathrm{NMR} \mathrm{(100} \mathrm{MHz,} \mathrm{CDCl}_{3}\right) \delta: 183.02,167.74$, $149.02,140.24,138.19,137.92,137.40,137.01,62.08$, 25.42, 22.39, 14.22; IR (film) v: 2984, 1725, 1619, 1584, $1499,1445,1373,1282,1265,1225,1171,1119,1023$, $996,897,861,730 \mathrm{~cm}^{-1}$. HRMS (ESI+) calcd for $\mathrm{C}_{12} \mathrm{H}_{14}-$ $\mathrm{ClO}_{3}[\mathrm{M}+\mathrm{H}]^{+}:$241.0626, found 241.0621.

3.2.54-溴-3,6-二甲基-7-氧代-1,3,5-环庚三烯-1-羒 酸乙酯 $(8)$ 的合成

将化合物 3 (200 mg, $0.90 \mathrm{mmol}$ )溶于二氯甲烷(3 $\mathrm{mL})$ 中, 然后加入 $\mathrm{PPh}_{3}(283 \mathrm{mg}, 1.08 \mathrm{mmol})$ 和 NBS (192 $\mathrm{mg}, 1.08 \mathrm{mmol})$. 室温搅拌 $1 \mathrm{~h}, \mathrm{TLC}$ 监测原料消耗完毕 后, 用 $\mathrm{Na}_{2} \mathrm{~S}_{2} \mathrm{O}_{3}$ 溶液淬灭. 分离有机相, 水相用二氯甲 烷萃取 $(5 \mathrm{~mL} \times 3)$, 合并有机相, 用饱和食盐水洗涤, 无 水 $\mathrm{Na}_{2} \mathrm{SO}_{4}$ 干燥后浓缩, 硅胶柱色谱法纯化 [ $V$ (石油醚) : $V($ 乙酸乙酯 $)=10 ： 1$ ]得到黄色油状液体 $8(241 \mathrm{mg}$, 产 率 94\%). ${ }^{1} \mathrm{H}$ NMR (400 MHz, $\left.\mathrm{CDCl}_{3}\right) \delta: 7.57$ (s, 1H), 7.39 
(s, 1H), 4.30 (q, J=7.1 Hz, 2H), $2.41(\mathrm{~s}, 3 \mathrm{H}), 2.24$ (s, 3H), $1.34 \sim 1.28(\mathrm{~m}, 3 \mathrm{H}) ;{ }^{13} \mathrm{C}$ NMR $\left(100 \mathrm{MHz}, \mathrm{CDCl}_{3}\right) \delta$ : $183.28,167.56,148.59,139.77,139.42,137.71,137.45$, 132.39, 61.98, 29.10, 22.12, 14.14; IR (film) v: 2982, 2729, 1723, 1615, 1579, 1494, 1443, 1371, 1273, 1220, 1172, 1114, 1020, 988, 897, 859, 803, 746, $718 \mathrm{~cm}^{-1}$. HRMS (ESI + ) calcd for $\mathrm{C}_{12} \mathrm{H}_{14} \mathrm{BrO}_{3}[\mathrm{M}+\mathrm{H}]^{+}: 285.0120$, found 285.0109 .

$3.2 .63,6$-二甲基-7-氧代-4-((三氟甲基)磺酰基)氧 基)-1,3,5-环庚三烯-1-羧酸乙酯(9)的合成

将化合物 3 (200 mg, $0.90 \mathrm{mmol})$ 溶于无水二氯甲烷 (3 mL)中, 体系冷却到 $-78{ }^{\circ} \mathrm{C}$ 左右加入三乙胺 $(109 \mathrm{mg}$, $1.08 \mathrm{mmol}$ ), 搅拌 $20 \mathrm{~min}$ 后, 滴入三氟甲磺酸酐(457 $\mathrm{mg}, 1.62 \mathrm{mmol})$, 在相同温度下搅拌 $1 \mathrm{~h}, \mathrm{TLC}$ 监测原料 消耗完后, 加水淬灭. 分离有机相, 水相用二氯甲烷萃 取 $(5 \mathrm{~mL} \times 3)$, 合并有机相, 用饱和食盐水洗涤, 无水 $\mathrm{Na}_{2} \mathrm{SO}_{4}$ 干燥后浓缩, 硅胶柱色谱法纯化 $[V$ (石油醚)： $V($ 乙酸乙酯 $)=10 ： 1]$ 得到黄色油状液体 $9(290 \mathrm{mg}$, 产 率为 95\%). ${ }^{1} \mathrm{H} \mathrm{NMR}\left(400 \mathrm{MHz}, \mathrm{CDCl}_{3}\right) \delta: 7.45(\mathrm{~s}, 1 \mathrm{H})$, $7.11(\mathrm{~s}, 1 \mathrm{H}), 4.35$ (q, $J=7.1 \mathrm{~Hz}, 2 \mathrm{H}), 2.39$ (s, 3H), 2.33 (s, $3 \mathrm{H}), 1.35(\mathrm{t}, J=7.1 \mathrm{~Hz}, 3 \mathrm{H}) ;{ }^{13} \mathrm{C} \mathrm{NMR}\left(100 \mathrm{MHz}, \mathrm{CDCl}_{3}\right)$ $\delta: 182.79,167.00,150.44,149.11,139.57,137.54,133.46$, $129.58,120.05,116.86,62.40,22.57,20.66,14.19$; IR (film) $v: 2986,1731,1643,1597,1524,1420,1377,1295$, 1213, 1171, 1137, 1026, 931, 874, $763 \mathrm{~cm}^{-1}$. HRMS $(\mathrm{ESI}+)$ calcd for $\mathrm{C}_{13} \mathrm{H}_{13} \mathrm{~F}_{3} \mathrm{O}_{6} \mathrm{SNa}[\mathrm{M}+\mathrm{Na}]^{+}: 377.0277$, found 377.0250 .

3.2.73,6-二甲基-7-氧代-4-乙烯基-1,3,5-环庚三烯1-羧酸乙酯 $(\mathbf{1 0})$ 的合成

将 $\mathrm{LiCl}$ (53 mg, $1.25 \mathrm{mmol})$ 和 $\mathrm{Pd}\left(\mathrm{PPh}_{3}\right)_{2} \mathrm{Cl}_{2}(5.50 \mathrm{mg}$, $0.008 \mathrm{mmol}$ )溶于无水 DMF (3 mL), 充分搅拌溶解后加 入化合物 9 (200 mg, $0.90 \mathrm{mmol})$, 搅拌几分钟后加入三 丁基乙烯基锡(186 mg, $0.58 \mathrm{mmol}$ ), $80{ }^{\circ} \mathrm{C}$ 下反应 $1.5 \mathrm{~h}$. 使用 TLC 监测原料消耗完后, 用 KF 溶液淬灭并搅拌 30 $\min$, 分离有机相, 水相用乙酸乙酯萃取 $(3 \mathrm{~mL} \times 3)$, 合 并有机相, 用饱和食盐水洗涤, 无水 $\mathrm{Na}_{2} \mathrm{SO}_{4}$ 干燥后浓 缩, 硅胶柱色谱法纯化 $[V$ (石油醚) : $V$ (乙酸乙酯 $)=20$ : 1]得到黄色固体 10 (45 mg, 产率 50\%). m.p. 48 50 ${ }^{\circ} \mathrm{C}$; ${ }^{1} \mathrm{H}$ NMR (400 MHz, $\mathrm{CDCl}_{3}$ ) $\delta: 7.43(\mathrm{~d}, J=10.6 \mathrm{~Hz}, 2 \mathrm{H})$, $6.84(\mathrm{dd}, J=17.3,11.1 \mathrm{~Hz}, 1 \mathrm{H}), 5.65$ (d, $J=17.4 \mathrm{~Hz}, 1 \mathrm{H})$, $5.50(\mathrm{~d}, J=11.3 \mathrm{~Hz}, 1 \mathrm{H}), 4.32$ (q, $J=7.1 \mathrm{~Hz}, 2 \mathrm{H}), 2.30$ (s, $6 \mathrm{H}), 1.33$ (t, $J=7.1 \mathrm{~Hz}, 3 \mathrm{H}) ;{ }^{13} \mathrm{C} \mathrm{NMR}\left(100 \mathrm{MHz}, \mathrm{CDCl}_{3}\right)$ $\delta: 183.72,168.25,149.21,141.76,140.74,138.19,137.13$, $136.98,134.44,119.92,61.79,23.58,23.03,14.20$; IR (film) $v: 3090,2980,1721,1598,1574,1497,1445,1414$,
$1372,1302,1231,1171,1117,1022,985,922,863,844$, $768,736 \mathrm{~cm}^{-1}$. HRMS (ESI+) calcd for $\mathrm{C}_{14} \mathrm{H}_{16} \mathrm{O}_{3} \mathrm{Na}$ $[\mathrm{M}+\mathrm{Na}]^{+}:$255.0991, found 255. 0987.

3.2.84-甲酰基-3,6-二甲基-7-氧代-1,3,5-环庚三烯1 -羧酸乙酯(11)的合成

将化合物 10 (70 mg, $0.30 \mathrm{mmol})$ 溶于丙酮 $(3 \mathrm{~mL})$ 、 水 $(1 \mathrm{~mL})$ 的混合溶液中, 充分搅拌溶解后加入 2,6 -二甲 基吡啶(33 mg, $0.30 \mathrm{mmol}$ )和 $\mathrm{NaIO}_{4}(105 \mathrm{mg}, 0.46 \mathrm{mmol}$ ), 室温摚拌 $5 \mathrm{~min}$, 加入 $\mathrm{K}_{2} \mathrm{OsO}_{4} \cdot 2 \mathrm{H}_{2} \mathrm{O}(5.2 \mathrm{mg}, 0.02$ $\mathrm{mmol})$, 室温下搅拌 $18 \mathrm{~h}$ 后. TLC 监测原料消耗完后, 用 $\mathrm{Na}_{2} \mathrm{~S}_{2} \mathrm{O}_{3}$ 溶液淬灭. 分离有机相, 水相用 EtOAc 萃取(3 $\mathrm{mL} \times 3)$, 合并有机相, 用饱和食盐水洗涤, 无水 $\mathrm{Na}_{2} \mathrm{SO}_{4}$ 干燥后浓缩, 硅胶柱色谱法纯化 $[V$ (石油醚) : $V($ 乙酸乙 酯 $)=10 ： 1$ 得到淡黄色固体 $\mathbf{1 1}(52 \mathrm{mg}$, 产率 75\%). m.p. $116 \sim 118{ }^{\circ} \mathrm{C} ;{ }^{1} \mathrm{H}$ NMR $\left(400 \mathrm{MHz}, \mathrm{CDCl}_{3}\right) \delta: 10.36(\mathrm{~s}$, $1 \mathrm{H}), 7.73$ (s, 1H), 7.42 (s, 1H), 4.35 (q, $J=7.1 \mathrm{~Hz}, 2 \mathrm{H})$, $2.68(\mathrm{~s}, 3 \mathrm{H}), 2.32(\mathrm{~s}, 3 \mathrm{H}), 1.35$ (t, $J=7.1 \mathrm{~Hz}, 3 \mathrm{H}) ;{ }^{13} \mathrm{C}$ NMR $\left(100 \mathrm{MHz}, \mathrm{CDCl}_{3}\right) \delta: 190.99,183.89,167.24$, $149.66,147.42,142.49,140.07,136.24,130.72,62.34$, 22.43, 20.81, 14.19; IR (film) v: 3359, 2924, 2853, 1728, $1685,1608,1589,1524,1445,1374,1301,1241,1149$, 1119, 1084, 1022, 940, 853, $782 \mathrm{~cm}^{-1}$. HRMS (ESI+) calcd for $\mathrm{C}_{13} \mathrm{H}_{14} \mathrm{O}_{4} \mathrm{Na}[\mathrm{M}+\mathrm{Na}]^{+}:$257.0784, found 257.0789 .

3.2.94-(羟甲基)-3,6-二甲基-7-氧代-1,3,5-环庚三烯1 -羧酸乙酯(12)的合成

将化合物 11 (100 mg, $0.43 \mathrm{mmol}$ )溶于 $\mathrm{MeOH}(2$ $\mathrm{mL}$ ), 然后加入四丁基嗍氢化铵(131 mg, $0.51 \mathrm{mmol}$ ). 室 温下搅拌 $10 \mathrm{~min}$, TLC 监测原料消耗完毕后, 用饱和 $\mathrm{NH}_{4} \mathrm{Cl}$ 溶液淬灭. 分离有机相, 水相用 EtOAc 萃取(1 $\mathrm{mL} \times 3)$, 合并有机相, 用饱和食盐水洗涤, 无水 $\mathrm{Na}_{2} \mathrm{SO}_{4}$ 干燥后浓缩, 硅胶柱色谱法纯化 $[V$ (石油醚) : $V($ 乙酸乙 酯 $)=5: 1$ ]得到白色固体 12 (73 mg, 产率 72\%). m.p. $97 \sim 100{ }^{\circ} \mathrm{C} ;{ }^{1} \mathrm{H}$ NMR (400 MHz, $\left.\mathrm{CDCl}_{3}\right) \delta: 7.63(\mathrm{~s}, 1 \mathrm{H})$, 7.30 (s, 1H), 4.51 (s, 2H), 4.33 (q, $J=7.1 \mathrm{~Hz}, 2 \mathrm{H}), 3.55$ (br, 1H), 2.25 (s, 3H), 2.20 (s, 3H), 1.34 (t, J=7.1 Hz, 3H); ${ }^{13} \mathrm{C}$ NMR $\left(100 \mathrm{MHz}, \mathrm{CDCl}_{3}\right) \delta: 183.02,168.71,150.12$, $146.45,140.79,139.35,138.39,136.31,64.47,62.08$, 23.03, 22.25, 14.22; IR (film) $v: 3417,2982,2926,1722$, $1609,1558,1506,1445,1372,1301,1237,1169,1122$, 1022, 912, 861, 775, $732 \mathrm{~cm}^{-1}$. HRMS (ESI+) calcd for $\mathrm{C}_{13} \mathrm{H}_{16} \mathrm{O}_{4} \mathrm{Na}[\mathrm{M}+\mathrm{Na}]^{+}: 259.0940$, found 259.0924.

3.2.104-(乙酰氧基甲基)-3,6-二甲基-7-氧代-1,3,5环庚三烯-1-羧酸乙酯(13)的合成

将化合物 12 (10 mg, $0.042 \mathrm{mmol}$ ) 溶于无水 $\mathrm{CH}_{2} \mathrm{Cl}_{2}$ 
$(2 \mathrm{~mL})$, 用冰水将体系温度降低至 $0{ }^{\circ} \mathrm{C}$ 左右. 滴加三乙 胺 $(8 \mathrm{mg}, 0.084 \mathrm{mmol})$, 搅拌 $5 \mathrm{~min}$ 后, 依次加入 4-二甲 氨基吡啶(DMAP) $\left(0.12 \mathrm{mg}, 0.001 \mathrm{mmol}\right.$ )和 $\mathrm{Ac}_{2} \mathrm{O}$ (4.8 $\mathrm{mg}, 0.046 \mathrm{mmol}), 0{ }^{\circ} \mathrm{C}$ 搅拌 $3 \mathrm{~h}$. TLC 监测原料消耗完后, 加水淬灭. 分离有机相, 水相用 $\mathrm{CH}_{2} \mathrm{Cl}_{2}$ 萃取 $(1 \mathrm{~mL} \times 3)$, 合并有机相, 用饱和食盐水洗涤, 无水 $\mathrm{Na}_{2} \mathrm{SO}_{4}$ 干燥后浓 缩, 硅胶柱色谱法纯化 $[V$ (石油醚) : $V$ (乙酸乙酯 $)=5$ : 1]得到淡黄色固体 13 (12 mg, 产率 96\%). m.p. 62 $65{ }^{\circ} \mathrm{C}$; ${ }^{1} \mathrm{H}$ NMR $\left(400 \mathrm{MHz}, \mathrm{CDCl}_{3}\right) \delta: 7.43$ (s, 1H), 7.36 (s, 1H), 4.99 (s, 2H), 4.36 (q, $J=7.1 \mathrm{~Hz}, 2 \mathrm{H}), 2.39$ (s, 3H), $2.32(\mathrm{~s}, 3 \mathrm{H}), 2.13(\mathrm{~s}, 3 \mathrm{H}), 1.36(\mathrm{t}, J=7.1 \mathrm{~Hz}, 3 \mathrm{H}) ;{ }^{13} \mathrm{C}$ NMR (100 MHz, $\left.\mathrm{CDCl}_{3}\right) \delta: 183.07,170.43,167.88$, $149.61,140.26,139.95,139.77,139.40,136.92,66.25$, 61.74, 22.71, 22.64, 20.71, 14.03; IR (film) v: 3057, 2985, $1728,1619,1580,1514,1444,1374,1300,1265,1221$, 1172, 1121, 1022, 971, 897, 853, $731 \mathrm{~cm}^{-1}$. HRMS (ESI + ) calcd for $\mathrm{C}_{15} \mathrm{H}_{18} \mathrm{O}_{5} \mathrm{Na}[\mathrm{M}+\mathrm{Na}]^{+}: 301.1046$, found 301.1036 .

\section{3 生物活性测试}

人胃癌细胞 MGC-27 从供应商 ATCC (American Type Culture Collection)购买, 然后在高葡萄糖 Dulbecco 改良的 Eagle 培养基(Sigma)中培养, 并添加了 10\%胎牛 血清(Gibco), $100 \mathrm{U} / \mathrm{mL}$ 青霉素和链霉素.

细胞存活率实验: 所有合成的化合物预先配成 DMSO 溶液 $\left(50 \mu \mathrm{mol} \cdot \mathrm{L}^{-1}\right)$, 纯 DMSO 作为空白对照, 细 胞存活率使用细胞计数试剂盒(CCK-8) (C0038, Beyotime Biotechnology), 根据制造商的说明进行, 处理时 间 $30 \mathrm{~h}$, 每组平行实验重复 3 次.

细胞调亡实验: 分别用纯 DMSO 和化合物 9 的 DMSO 溶液 $\left(50 \mu \mathrm{mol} \cdot \mathrm{L}^{-1}\right)$ 处理人胃癌细胞 MGC-27, 处 理时间 $30 \mathrm{~h}$, 最后通过流式细胞术检测了细胞淍亡情 况, 每组平行实验重复 3 次.

辅助材料(Supporting Information) 化合物 2, 3, 5 13 的 ${ }^{1} \mathrm{H}$ NMR 和 ${ }^{13} \mathrm{C}$ NMR 谱图, 以及化合物 6 和 13 的单 晶衍射数据. 这些材料可以免费从本刊网站(http://siocjournal.cn/)上下载.

\section{References}

[1] Liu, N.; Song, W.; Schienebeck, C. M.; Zhang, M.; Tang, W. Tetrahedron 2014, 70, 9281.

[2] Zhao, J. Curr. Med. Chem. 2007, 14, 2597.

[3] Rabe, P.; Klapschinski, T. A.; Brock, N. L.; Citron, C. A.; D'Alvise, P.; Gram, L.; Dickschat, J. S. Beilstein J. Org. Chem. 2014, 10, 1796.

[4] Cao, F.; Orth, C.; Donlin, M. J.; Adegboyega, P.; Meyers, M. J.; Murelli, R. P.; Elagawany, M.; Elgendy, B.; Tavis, J. E. ACS Omega 2018, 3, 15125 .
[5] Pietra, F. Chem. Rev. 2002, 73, 293.

[6] Pauson, P. L. Chem. Rev. 1955, 55, 9.

[7] Okamura, H.; Iiji, H.; Hamada, T.; Iwagawa, T.; Furuno, H. Tetrahedron 2009, 65, 10709.

[8] Trost, B. M.; McDougall, P. J.; Hartmann, O.; Wathen, P. T. J. Am. Chem. Soc. 2008, 130, 14960.

[9] Tejero, R.; Ponce, A.; Adrio, J.; Carretero, J. C. Chem. Commun. 2013, 49, 10406.

[10] Xie, M.; Liu, X.; Wu, X.; Cai, Y.; Lin, L.; Feng, X. Angew. Chem. Int. Ed. 2013, 52, 5604.

[11] Mose, R.; Preegel, G.; Larsen, J.; Jakobsen, S.; Iversen, E. H.; Jorgensen, K. A. Nat. Chem. 2017, 9, 487.

[12] Isakovic, L.; Ashenhurst, J. A.; Gleason, J. L. Org. Lett. 2001, 3 , 4189.

[13] Li, P.; Yamamoto, H. Chem. Commun. 2010, 46. 6294.

[14] Trust, T. J.; Bartlett, K. H. Antimicrob. Agents Chemother. 1975, 8, 381.

[15] Zhao, C.-X.; Li, B.-Q.; Shao, Z.-X.; Li, D.-H.; Jing, Y.-K.; Li, Z.-L.; Hua, H.-M. Tetrahedron Lett. 2019, 60. 151154.

[16] Xu, J.-B.; Fan, Y.-Y.; Gan, L.-S.; Zhou, Y.-B.; Li, J.; Yue, J.-M. Chem.-Eur. J. 2016, 22, 14648.

[17] Du, J.; Chiu, M.-H.; Nie, R.-L. J. Nat. Prod. 1999, 62, 1664.

[18] Yoon, K. D.; Jeong, D. G.; Hwang, Y. H.; Ryu, J. M.; Kim, J. J. Nat. Prod. 2007, 70, 2029.

[19] Ni, L.; Zhong, X.-H.; Chen, X.-J.; Zhang, B.-J.; Bao, M.-F.; Cai, X.-H. Phytochemistry 2018, 151, 50

[20] Li, Y.; Wang, Y.; Shao, Z.; Zhao, C.; Jing, Q.; Li, D.; Lin, B.; Jing, Y. Bioorg. Chem. 2020, 103, 104226.

[21] Buta, J. G.; Flippen, J. L.; Lusby, W. R. J. Org. Chem. 2002, 43, 1002.

[22] Zhang, M.; Liu, N.; Tang, W. J. Am. Chem. Soc. 2013, 135, 12434.

[23] Hegde, V.; Campitelli, M.; Quinn, R. J.; Camp, D. Org. Biomol. Chem. 2011, 9, 4570

[24] Ge, Z.-P.; Liu, H.-C.; Wang, G.-C.; Liu, Q.-F.; Xu, C.-H.; Ding, J.; Fan, Y.-Y.; Yue, J.-M. J. Nat. Prod. 2019, 82, 1565.

[25] Fan, Y.-Y.; Xu, J.-B.; Liu, H.-C.; Gan, L.-S.; Ding, J.; Yue, J.-M. J. Nat. Prod. 2017, 80, 3159

[26] Zhao, J.-X.; Fan, Y.-Y.; Xu, J.-B.; Gan, L.-S.; Xu, C.-H.; Ding, J.; Yue, J.-M. J. Nat. Prod. 2017, 80, 356.

[27] Morita, Y.; Matsumura, E.; Tsujibo, H.; Yasuda, M.; Okabe, T.; Sakagami, Y.; Kumeda, Y.; Ishida, N.; Inamor, Y. Biol. Pharm. Bull. 2002, 25, 981 .

[28] Hong, S. K.; Kim, H.; Seo, Y.; Lee, S. H.; Cha, J. K.; Kim, Y. G. Org. Lett. 2010, 12, 3954.

[29] Feldman, K. S.; Cutarelli, T. D.; Di Florio, R. J. Org. Chem. 2002, $67,8528$.

[30] Feldman, K. S.; Cutarelli, T. D. J. Am. Chem. Soc. 2002, 124, 11600.

[31] Zweig, M. H.; Chignell, C. F. Biochem. Pharmacol. 1973, 22, 2141.

[32] Chaudhuri, A. R.; Seetharamalu, P.; Schwarz, P. M.; Hausheer, F. H.; Ludueña, R. F. J. Mol. Biol. 2000, 303, 679.

[33] Zhang, H.-J.; Hu, L.; Ma, Z.; Li, R.; Zhang, Z.; Tao, C.; Cheng, B.; Li, Y.; Wang, H.-F.; Zhai, H.-B. Angew. Chem., Int. Ed. 2016, 55, 11638.

[34] Ma, Z.; Cheng, B.; Zhai, H. Asian J. Org. Chem. 2014, 3, 1097.

[35] Evanno, L.; Jossang, A.; Nguyen-Pouplin, J.; Delaroche, D.; Herson, P.; Seuleiman, M.; Bodo, B.; Nay, B. Planta Med. 2008, 74, 870 .

[36] Yamato, M.; Hashigaki, K.; Ishikawa, S.; Kokubu, N.; Inoue, Y.; Tsuruo, T.; Tashiro, T. J. Med. Chem. 2002, 28, 1026.

[37] Yamato, M.; Hashigaki, K.; Kokubu, N.; Tashiro, T.; Tsuruo, T. J. Med. Chem. 1986, 29, 1202.

[38] Yamato, M.; Hashigaki, K.; Kokubu, N.; Tsuruo, T.; Tashiro, T. J. Med. Chem. 1984, 27, 1749 .

[39] Yamato, M.; Hashigaki, K.; Sakai, J.; Takeuchi, Y.; Tsukagoshi, S.; Tashiro, T.; Tsuruo, T. J. Med. Chem. 1987, 30, 1245.

[40] Bagli, J. F.; Bogri, T.; Palameta, B.; Martel, R.; Robinson, W.; Pugsley, T.; Lippmann, W. J. Med. Chem. 2002, 22, 1186. 
[41] Fitzmaurice, C.; Dicker, D.; Pain, A.; Hamavid, H.; Moradi-Lakeh, M. MacIntyre, M. F.; Allen, C.; Hansen, G. JAMA Oncol. 2015, 1, 505.

[42] Torre, L. A.; Bray, F.; Siegel, R. L.; Ferlay, J.; Lortet-Tieulent, J.; Jemal, A. Cancer J. Clin. 2015, 65, 87.

[43] Huang, L.; Qi, D.-J.; He, W.; Xu, A.-M. Oncotarget 2017, 8, 70332.

[44] Chen, Z.-M.; Huang, L.; Li, M.-M.; Meng, L.; Ying, S.-C.; Xu, A.-M. Sci. Rep. 2018, 8, 9307.

[45] Allemani, C.; Weir, H. K.; Carreira, H.; Harewood, R.; Spika, D.; Wang, X.-S.; Bannon, F.; Ahn, J. V. Lancet 2015, 385, 977.

[46] Van Cutsem, E.; Sagaert, X.; Topal, B.; Haustermans, K.; Prenen, H. Lancet 2016, 388, 2654.

[47] Dauben, W. G.; Eastham, J. F. J. Am. Chem. Soc. 1950, 72, 2305.

[48] Ter Borg, A. P.; van Helden, R.; Bickel, A. F.; Renold, W.; Dreiding, A. S. Helv. Chim. Acta 1960, 43, 457.
[49] Nicolaou, K. C.; Zhong, Y.-L. Baran, P. S. J. Am. Chem. Soc. 2000, 122,7596

[50] Arican, D.; Brückner, R. Org. Lett. 2013, 15, 2582.

[51] Harmata, M. Chem. Commun. 2010, 46. 8886.

[52] Lohse, A. G.; Hsung, R. P. Chem.-Eur. J. 2011, 17, 3812.

[53] Harmata, M. Chem. Commun. 2010, 46, 8886.

[54] Ylijoki, K. E.; Stryker, J. M. Chem. Rev. 2013, 113, 2244.

[55] Pellissier, H. Adv. Synth. Catal. 2011, 353, 189.

[56] Butenschon, H. Angew. Chem., Int. Ed. 2008, 47, 5287.

[57] Zhao, J.-D.; Liu, J.; Xie, X.; Li, S.; Liu, Y.-H. Org. Lett. 2015, 17, 5926.

[58] Chen, M.; Chen, Y.; Sun, N.; Zhao, J.; Liu, Y.-H.; Li, Y. Angew. Chem., Int. Ed. 2015, 54, 1200.

[59] Chen, M.; Sun, N.; Xu, W.; Zhao, J.; Wang, G.; Liu, Y.-H. Chem.Eur. J. 2015, 21, 18571.

(Li, L.; Fan, Y.) 